\title{
Genome editing shows promise in an in vivo model of Duchenne muscular dystrophy
}

CRISPR-Cas9-based genome editing can restore dystrophin protein expression and improve muscle structure and function in the $m d x$ mouse model of Duchenne muscular dystrophy (DMD), according to three reports recently published in Science.

Most cases of DMD are caused by frameshift mutations in the dystrophin $(D M D)$ gene that lead to the production of a truncated, non-functional form of the dystrophin protein. The genome editing strategy is based on the premise that excision of one or more exons of the reading frame, enabling an internally truncated but functional protein to be generated.

Charles Gersbach and colleagues, who conducted one of the new studies, had previously demonstrated the efficacy of the CRISPR-Cas9mediated approach in cultured muscle cells from patients with DMD. "The next major step was to develop a way to correct the gene in muscle tissue in vivo," says Gersbach. "We needed to redesign our approach to target the mutation in the $m d x$ mouse model, develop a delivery strategy, and switch to an alternative CRISPR-Cas9 system that is compatible with that delivery strategy."

Gersbach's team constructed an adeno-associated virus (AAV)-based vector containing Cas9-based genome editing could address up to $83 \%$ of ... disease-causing mutations in ... DMD 5y an expression cassette for guide RNAs (gRNAs) that directed the Cas 9 endonuclease to excise exon 23 of the Dmd gene - the region that carries the disease-causing mutation in $m d x$ mice. "We used AAV because it is non-pathogenic and particularly effective at delivery to skeletal and cardiac muscle, and mutant gene can restore the DMD

has been demonstrated to be safe and effective in human clinical trials," explains Gersbach.

The gRNA-expressing vector was co-injected with a Cas9-expressing $\mathrm{AAV}$ vector into the tibialis anterior muscle in $m d x$ mice. Treated muscle tissue showed improved morphology and reduced inflammation compared with untreated muscle. In addition, twitch and tetanic forces, as well as resistance to fatigue produced by eccentric contractions, were enhanced in the treated muscle.

The authors also discovered that systemic gRNA-Cas9 delivery could rescue dystrophin expression in cardiac muscle in perinatal and 6-week-old $m d x$ mice. The ability to preserve cardiac muscle function is particularly important, as heart failure is a common cause of premature death in patients with DMD.

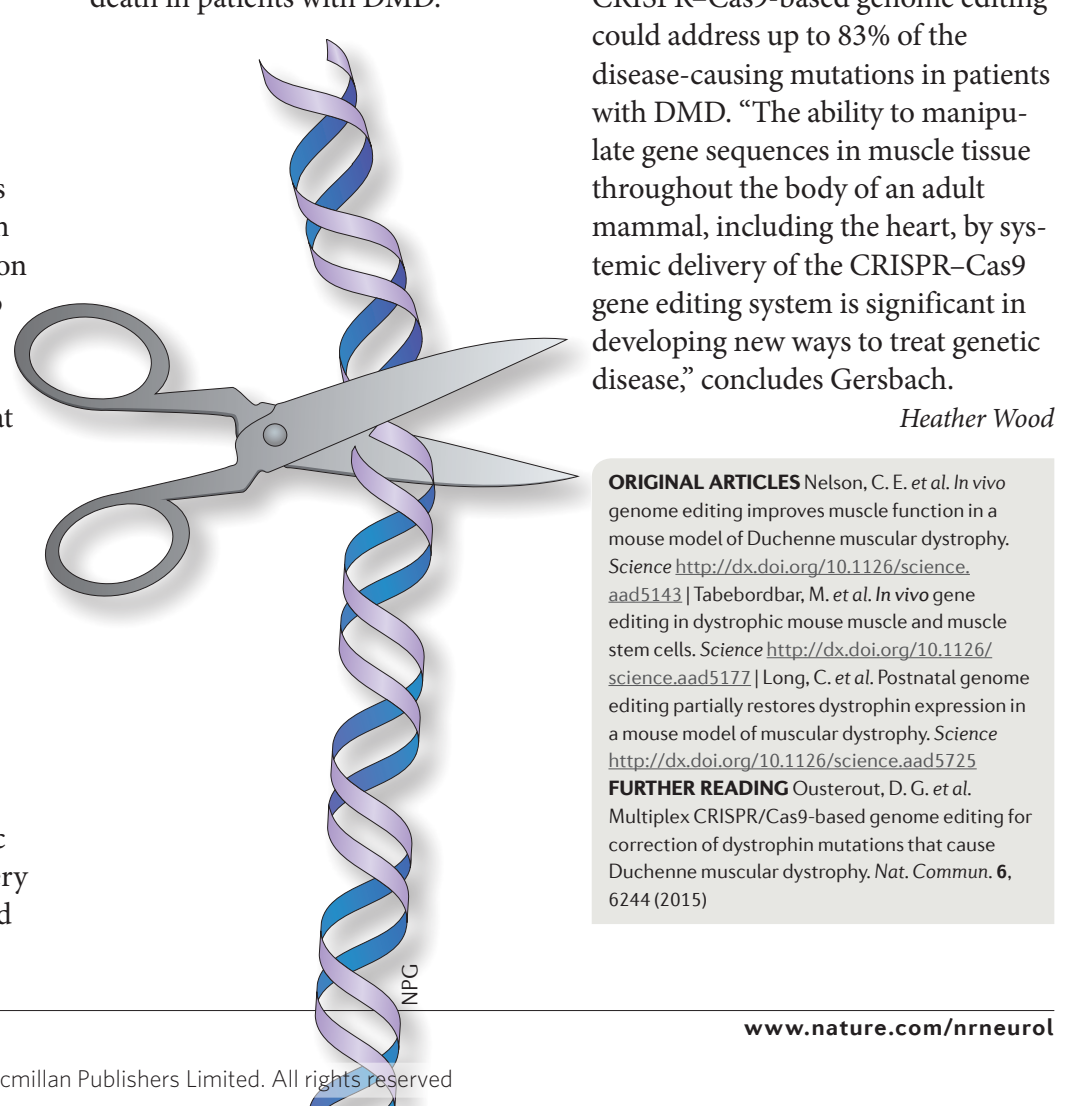

Using a similar approach, Amy Wagers and colleagues found that CRISPR-Cas9-mediated excision of exon 23 restored the Dmd reading frame in myofibres, cardiomyocytes and muscle stem cells in $m d x$ mice. Again, these effects were shown to translate into measurable amelioration of dystrophic pathology and muscle weakness.

The efficacy of the CRISPR-Cas9 approach was further confirmed in a third report from Eric Olson and colleagues. This group tested different routes of delivery for the AAV vectors, and they found that intraperitoneal, intramuscular and retro-orbital injections were all able to restore dystrophin expression and improve skeletal muscle function in postnatal $m d x$ mice.

Estimates indicate that CRISPR-Cas9-based genome editing could address up to $83 \%$ of the disease-causing mutations in patients with DMD. "The ability to manipuate gene sequences in muscle tissue mmal, including the heart, by systemic delivery of the CRISPR-Cas9 gene editing system is significant in developing new ways to treat genetic 